\title{
On the Matsumoto zeta-function
}

\author{
by
}

\section{A. LAURINČIKAS (Vilnius)}

1. Introduction. Let, as usual, $\mathbb{N}$ and $\mathbb{C}$ denote the sets of natural numbers and complex numbers, respectively. For $m \in \mathbb{N}$ let $g(m) \in \mathbb{N}$, $f(j, m) \in \mathbb{N}$, and $a_{m}^{(j)} \in \mathbb{C}, 1 \leq j \leq g(m)$, and define the polynomials of degree $f(1, m)+\ldots+f(g(m), m)$

$$
A_{m}(x)=\prod_{j=1}^{g(m)}\left(1-a_{m}^{(j)} x^{f(j, m)}\right) .
$$

Let $s=\sigma+i t$ be a complex variable and let $p_{m}$ denote the $m$ th prime number. In [10] K. Matsumoto introduced and considered the zeta-function $\varphi(s)$ given by

$$
\varphi(s)=\prod_{m=1}^{\infty} A_{m}^{-1}\left(p_{m}^{-s}\right) .
$$

Denote by meas $\{A\}$ the Lebesgue measure of the set $A$, and let, for $T>0$,

$$
\nu_{T}^{t}(\ldots)=\frac{1}{T} \operatorname{meas}\{t \in[0, T]: \ldots\}
$$

where instead of dots we write a condition satisfied by $t$. Assuming the conditions

$$
g(m) \leq c_{1} p_{m}^{\alpha}, \quad\left|a_{m}^{(j)}\right| \leq p_{m}^{\beta}
$$

with a positive constant $c_{1}$ and non-negative constants $\alpha$ and $\beta$, K. Matsumoto [10] proved two limit theorems for $\log \varphi(s)$ in the complex plane. Note that under the condition (1) the product in the definition of $\varphi(s)$ converges absolutely for $\sigma>\alpha+\beta+1$ and defines a holomorphic function with no zeros.

The first theorem from [10] considers the case $\sigma>\alpha+\beta+1$. Let

$$
V(T, R)=\nu_{T}^{t}\left(\log \varphi\left(\sigma_{0}+i t\right) \in R\right)
$$

1991 Mathematics Subject Classification: Primary 11M41.

Partially supported by Grant from Lithuanian Foundation of Studies and Science. 
where $R$ is a closed rectangle in $\mathbb{C}$ with edges parallel to the axes, and $\log \varphi\left(\sigma_{0}+i t\right)$ is the following sum of principal values:

$$
\log \varphi\left(\sigma_{0}+i t\right)=-\sum_{m=1}^{\infty} \sum_{j=1}^{g(m)} \log \left(1-a_{m}^{(j)} p_{m}^{-f(j, m)\left(\sigma_{0}+i t\right)}\right) .
$$

Theorem 1 (K. Matsumoto, [10]). Let $\sigma_{0}>\alpha+\beta+1$. Under the condition (1) the following limit exists:

$$
V\left(R ; \sigma_{0}\right)=\lim _{T \rightarrow \infty} V(T, R) .
$$

The second theorem of [10] deals with the case $\sigma<\alpha+\beta+1$. Let $\varrho$ be a constant with $\alpha+\beta+1 / 2 \leq \varrho<\alpha+\beta+1$, and assume that $\varphi(s)$ can be meromorphically continued to the region $\sigma \geq \varrho$. Moreover, let $\mathcal{P}$ be the set of all possible zeros and poles of $\varphi(s)$ in the strip $\varrho \leq \sigma \leq \alpha+\beta+1$, and

$$
G=\{s \in \mathbb{C}: \sigma>\varrho\}-\bigcup_{s^{\prime}=\sigma^{\prime}+i t^{\prime} \in \mathcal{P}}\left\{s=\sigma+i t^{\prime}: \varrho \leq \sigma \leq \sigma^{\prime}\right\} .
$$

For $s_{0}=\sigma_{0}+i t_{0} \in G$ the value of $\log \varphi\left(s_{0}\right)$ is defined by analytic continuation along the path $\left\{s=\sigma+i t_{0}: \sigma \geq \sigma_{0}\right\}$. Now let $\varrho<\sigma_{0} \leq \alpha+\beta+1$, and

$$
W(T, R)=\nu_{T}^{t}\left(\sigma_{0}+i t \in G, \log \varphi\left(\sigma_{0}+i t\right) \in R\right) .
$$

Theorem 2 (K. Matsumoto, [10]). Let the following conditions be satisfied:

(i) the condition (1);

(ii) the function $\varphi(s)$ is meromorphic in the half-plane $\sigma \geq \varrho$, all poles in this region are included in a compact set, and there is no pole on the line $\sigma=\varrho$

(iii) for $\sigma \geq \varrho$,

$$
\varphi(\sigma+i t)=B|t|^{c_{2}}
$$

(iv) we have the estimate

$$
\int_{0}^{T}|\varphi(\varrho+i t)|^{2} d t=B T .
$$

Then, for any $\sigma_{0}>\varrho$, the following limit exists:

$$
W\left(R ; \sigma_{0}\right)=\lim _{T \rightarrow \infty} W(T, R) .
$$

Here and in what follows $B$ denotes a number (not always the same) bounded by a constant, and $c_{2}, c_{3}, \ldots$ are positive constants.

Note that, in fact, Theorems 1 and 2 are limit theorems in the sense of the weak convergence of probability measures in $\mathbb{C}$. 
In [11] and [12] K. Matsumoto himself and in [3] jointly with T. Hattori treated the limit measure of Theorem 2 proving some precise upper and lower bounds for it.

The function $\varphi(s)$ is a generalization of Dirichlet series attached to certain cusp forms and also of the Dedekind zeta-function of algebraic number fields. Limit theorems for these functions were obtained in [9] and [13], respectively.

In [4] we generalized Theorems 1 and 2 proving two functional limit theorems with a weight for the function $\varphi(s)$.

Let $\mathcal{G}$ be a region in the complex plane. Denote by $H(\mathcal{G})$ the space of functions analytic on $\mathcal{G}$, equipped with the topology of uniform convergence on compacta. Moreover, let $T_{0}$ be a fixed positive number, and let $w(\tau)$ be a positive function of bounded variation on $\left[T_{0}, \infty\right)$. Set

$$
U=U(T, w)=\int_{T_{0}}^{T} w(\tau) d \tau
$$

and suppose that $\lim _{T \rightarrow \infty} U(T, w)=\infty$. Moreover, let $\mathcal{B}(S)$ stand for the class of Borel subsets of the space $S$, and let $D_{1}=\{s \in \mathbb{C}: \sigma>\alpha+\beta+1\}$. Define the probability measure

$$
P_{T, w}(A)=\frac{1}{U} \int_{T_{0}}^{T} w(\tau) I_{\{\tau: \varphi(s+i \tau) \in A\}} d \tau, \quad A \in \mathcal{B}\left(H\left(D_{1}\right)\right),
$$

where $I_{A}$ denotes the indicator function of the set $A$.

TheOREM 3 [4]. Under the condition (1) there exists a probability measure $P_{w}$ on $\left(H\left(D_{1}\right), \mathcal{B}\left(H\left(D_{1}\right)\right)\right)$ such that the measure $P_{T, w}$ converges weakly to $P_{w}$ as $T \rightarrow \infty$.

Now let $D_{2}=\left\{s \in \mathbb{C}: \sigma>\varrho_{0}\right\}$ where $\alpha+\beta+1 / 2 \leq \varrho_{0}<\alpha+\beta+1$. Denote by $M\left(D_{2}\right)$ the space of functions meromorphic on $D_{2}$ equipped with the topology of uniform convergence on compacta and suppose that for the functions $w(\tau)$ and $\varphi(s)$ the estimate

$$
\int_{T_{0}}^{T} w(\tau)|\varphi(\sigma+i \tau+i t)|^{2} d \tau=B U(1+|t|)
$$

holds for $\sigma>\varrho_{0}$ and for all real $t$. Define the probability measure

$$
Q_{T, w}(A)=\frac{1}{U} \int_{T_{0}}^{T} w(\tau) I_{\{\tau: \varphi(s+i \tau) \in A\}} d \tau, \quad A \in \mathcal{B}\left(M\left(D_{2}\right)\right) .
$$

TheOREM 4 [4]. Let the function $\varphi(s)$ be meromorphic in the half-plane $\sigma>\varrho_{0}$. Suppose that all poles in this region are included in a compact set. Then under the conditions (1), (2) and (iii), (iv) of Theorem 2 there exists a 
probability measure $Q_{w}$ on $\left(M\left(D_{2}\right), \mathcal{B}\left(M\left(D_{2}\right)\right)\right)$ such that the measure $Q_{T, w}$ converges weakly to $Q_{w}$ as $T \rightarrow \infty$.

In [5] and [6] we indicated the explicit form of the limit measures in Theorems 3 and 4 , respectively.

Denote by $\gamma$ the unit circle on complex plane, and let

$$
\Omega=\prod_{p} \gamma_{p}
$$

where $\gamma_{p}=\gamma$ for all primes $p$. With the product topology and pointwise multiplication the infinite-dimensional torus $\Omega$ is a compact topological group. Therefore there exists the probability Haar measure $m$ on $(\Omega, \mathcal{B}(\Omega))$. Thus we obtain a probability space $(\Omega, \mathcal{B}(\Omega), m)$. Let $\omega(p)$ stand for the projection of $\omega \in \Omega$ onto the coordinate space $\gamma_{p}$. Then, taking

$$
\omega(k)=\prod_{p^{r} \| k} \omega^{r}(p)
$$

where $p^{r} \| k$ means that $p^{r} \mid k$ but $p^{r+1} \nmid k$, we obtain an extension of the function $\omega(p)$ to the set $\mathbb{N}$ as a completely multiplicative unimodular function.

Let $D=\{s \in \mathbb{C}: \sigma>\alpha+\beta+1 / 2\}$, and set, for $s \in D$ and $\omega \in \Omega$,

$$
\varphi(s, \omega)=\sum_{k=1}^{\infty} \frac{b(k) \omega(k)}{k^{s}},
$$

where, for $\sigma>\alpha+\beta+1$,

$$
\varphi(s)=\sum_{k=1}^{\infty} \frac{b(k)}{k^{s}} .
$$

LEMMA 1. The series

$$
\sum_{k=1}^{\infty} \frac{b(k) \omega(k)}{k^{s}}
$$

converges uniformly on compact subsets of $D$ for m-almost all $\omega \in \Omega$.

Proof. Since $b(k)=B k^{\alpha+\beta+\varepsilon}[10]$, we see that, as $N \rightarrow \infty$,

$$
\sum_{k \leq N}|b(k)|^{2}=B N^{2(\alpha+\beta+\varepsilon+1 / 2)}
$$

Therefore, the lemma is a consequence of Lemma 3.4.3 from [1]. See also Lemma 5.1.6 of [7].

Lemma 1 shows that $\varphi(s, \omega)$ is an $H(D)$-valued random element defined on the probability space $(\Omega, \mathcal{B}(\Omega), m)$. Let $P_{\varphi}$ denote the distribution of $\varphi(s, \omega)$, and let $P_{j, \varphi}$ be the restriction of $P_{\varphi}$ to $\left(H\left(D_{j}\right), \mathcal{B}\left(H\left(D_{j}\right)\right)\right), j=1,2$. 
Denote by $E \xi$ the mean of the random variable $\xi$. Let $X(\tau, \omega), \omega \in \Omega_{1}$, $\tau \in \mathbb{R}$, be an ergodic process, $E|X(\tau, \omega)|<\infty$, with sample paths almost surely integrable in the Riemann sense over every finite interval. Suppose that the function $w(\tau)$ satisfies

$$
\frac{1}{U} \int_{T_{0}}^{T} w(\tau) X(\tau, \omega) d \tau=E X(0, \omega)+o(1)
$$

almost surely as $T \rightarrow \infty$.

Theorem 5 [5]. Under the conditions (1) and (3) the measure $P_{w}$ in Theorem 3 coincides with $P_{1, \varphi}$.

Now suppose that the function $w(\tau)$ satisfies

$$
\frac{1}{U} \int_{T_{0}}^{T} w(\tau) X(t+\tau, \omega) d \tau=E X(0, \omega)+o(1+|t|)^{c_{3}}
$$

almost surely for all $t \in \mathbb{R}$ with some $c_{3}>0$ as $T \rightarrow \infty$.

TheOREM 6 [6]. Under the conditions of Theorem 4 and (4) the measure $Q_{w}$ in Theorem 4 coincides with $P_{2, \varphi}$.

Examples of functions $w(\tau)$ are given in [8].

The aim of this note is to obtain the universality property for the function $\varphi(s)$. Note that S. M. Voronin was the first who proved the universality of the Riemann zeta-function [15]. Some new versions of the universality of functions given by Dirichlet series were proposed in [1] and [2]. In the sequel, we suppose that the function $\varphi(s)$ is analytic in the strip $D_{3}=\{s \in \mathbb{C}$ : $\left.\varrho_{0}<\sigma<\alpha+\beta+1\right\}$ where $\alpha+\beta+1 / 2 \leq \varrho_{0}<\alpha+\beta+1$. Moreover, we assume that

$$
M(m):=\left|\sum_{\substack{j=1 \\ f(j, m)=1}}^{g(m)} a_{m}^{(j)}\right| p_{m}^{-\alpha-\beta} \geq c_{4}>0
$$

for $m \geq 1$.

TheOREM 7. Let the conditions (1), (2), (iii) and (iv) of Theorem 2, and (4), (5) be satisfied. Let $K$ be a compact subset of the strip $D_{3}$ with connected complement. Let $f(s)$ be a non-vanishing continuous function on $K$ which is analytic in the interior of $K$. Then for every $\varepsilon>0$,

$$
\liminf _{T \rightarrow \infty} \frac{1}{U} \int_{T_{0}}^{T} w(\tau) I_{\left\{\tau: \sup _{s \in K}|\varphi(s+i \tau)-f(s)|<\varepsilon\right\}} d \tau>0 .
$$

The universality is an interesting and deep property of Dirichlet series. There exists a conjecture of Prof. I. A. Ibragimov that all Dirichlet series 
have the universality property. However, we think that the conjecture is too bold, because the proof of the universality of given Dirichlet series is usually complicated, and there are Dirichlet series for which the known methods are not applicable. On the other hand, all classical zeta-functions (the Riemann zeta-function, Dirichlet $L$-functions, Dedekind zeta-functions) are universal. This suggests searching for new classes of functions with the universality property. The condition (5) is, of course, sufficiently strong, which corresponds to the depth of the universality. The classical functions usually have other good properties helpful in proving their universality.

EXAMPLE. Let, for $\sigma>1$,

$$
\zeta_{a}(s)=\prod_{p}\left(1-\frac{a(p)}{p^{s}}\right)^{-1},
$$

where $a(m)$ is a completely multiplicative function such that $0<c_{5} \leq$ $|a(m)| \leq 1$, and $p$ denotes a prime number. It is not difficult to indicate some conditions for the analytic continuation of $\zeta_{a}(s)$ to the strip $1 / 2<\varrho<\sigma<1$ and for the validity of conditions (iii) and (iv) of Theorem 2. For example, it suffices to require some regularity condition for

$$
\sum_{m \leq x} a(m), \quad x \rightarrow \infty .
$$

The details can be found in [7].

The aim of this note is a generalization of the latter example. The Euler product of this example has only a linear factor for each prime. But Theorem 7 shows under the condition (5) the universality for the Euler product which has a polynomial factor of arbitrarily high degree for each prime. Moreover, in Theorem 7 a weight function is involved. Therefore Theorem 7 gives a generalization of the Example.

Denoting by $p$, as above, a prime number, we may change products and sums over $m$ to those over $p$. The condition (5) can be replaced by the following one: for every $\varepsilon>0$ and $x \rightarrow \infty$,

$$
\frac{1}{\pi(x)} \sum_{\substack{p \leq x \\ M(p)<\varepsilon}} 1=B x^{-\delta},
$$

where $\delta \geq 1 / 2$, and $\pi(x)$, as usual, denotes the number of primes not exceeding $x$.

REMARK. Theorem 7 is true with the condition (6) instead of (5).

2. A limit theorem in the space of analytic functions. In order to prove Theorem 7 first we must prove a limit theorem for the function $\varphi(s)$ 
in the space of functions analytic on $D_{3}$. To state such a theorem we will give some lemmas.

Lemma 2. For almost all $\omega \in \Omega$, the product

$$
\prod_{m=1}^{\infty} \prod_{j=1}^{g(m)}\left(1-\frac{\omega^{f(j, m)}\left(p_{m}\right) a_{m}^{(j)}}{p_{m}^{s f(j, m)}}\right)^{-1}
$$

converges uniformly on compact subsets of $D$, and

$$
\sum_{k=1}^{\infty} \frac{b(k) \omega(k)}{k^{s}}=\prod_{m=1}^{\infty} \prod_{j=1}^{g(m)}\left(1-\frac{\omega^{f(j, m)}\left(p_{m}\right) a_{m}^{(j)}}{p_{m}^{s f(j, m)}}\right)^{-1} .
$$

Proof. The lemma is a generalization of a similar result from [1]; see also Theorem 5.1 .7 of [7].

Since $\left|\omega\left(p_{m}\right)\right|=1$, for $\sigma>\alpha+\beta+1$, both the series and the product converge absolutely for any $\omega \in \Omega$, and thus the equality of the lemma holds. By Lemma 1 the series of the lemma converges uniformly for almost all $\omega \in \Omega$ on compact subsets of $D$. Therefore by analytic continuation it remains to show that the product of the lemma converges uniformly for almost all $\omega \in \Omega$ on compact subsets of $D$.

We can write the product in the form

$$
\prod_{m=1}^{\infty}\left(1+\sum_{j=1}^{g(m)} \frac{\omega^{f(j, m)}\left(p_{m}\right) a_{m}^{(j)}}{p_{m}^{s f(j, m)}}+r_{m}(s, \omega)\right),
$$

where the series $\sum_{m=1}^{\infty} r_{m}(s, \omega)$ converges uniformly on compact subsets of $D$. Thus it remains to prove that the series

$$
\sum_{m=1}^{\infty} \sum_{j=1}^{g(m)} \frac{\omega^{f(j, m)}\left(p_{m}\right) a_{m}^{(j)}}{p_{m}^{s f(j, m)}}
$$

converges uniformly for almost all $\omega \in \Omega$ on compact subsets of $D$. Clearly, the study of the latter series can be replaced by that of the series

$$
\sum_{m=1}^{\infty} \sum_{\substack{j=1 \\ f(j, m)=1}}^{g(m)} \frac{\omega\left(p_{m}\right) a_{m}^{(j)}}{p_{m}^{s}}=\sum_{m=1}^{\infty} \frac{\omega\left(p_{m}\right)}{p_{m}^{s}} \sum_{\substack{j=1 \\ f(j, m)=1}}^{g(m)} a_{m}^{(j)} .
$$

Let

$$
y_{m}(s, \omega)=\frac{\omega\left(p_{m}\right)}{p_{m}^{s}} \sum_{\substack{j=1 \\ f(j, m)=1}}^{g(m)} a_{m}^{(j)} .
$$

Since $\omega\left(p_{m}\right), m \in \mathbb{N}$, is a sequence of independent random variables (see $[7])$, we see that $y_{m}(s, \omega), m \in \mathbb{N}$, is a sequence of independent $H(D)$-valued 
random elements. It follows from the definition of $\omega\left(p_{m}\right)$ that $E y_{m}(s, \cdot)=0$ for all $m \in \mathbb{N}$. Moreover, for $m \in \mathbb{N}$,

$$
E\left|y_{m}(s, \cdot)\right|^{2} \leq \frac{1}{p_{m}^{2 \sigma-2 \alpha-2 \beta}},
$$

and thus

$$
\sum_{m=1}^{\infty} E\left|y_{m}(s, \cdot)\right|^{2}<\infty
$$

for $s \in D$. Hence by the Kolmogorov three series theorem (see, for example, [7], Theorem 1.2.11) the series (7) converges for almost all $\omega \in \Omega$ for each fixed $s \in D$. But if the Dirichlet series converges at the point $s_{0}=\sigma_{0}+i t_{0}$, then it converges uniformly on compacta in the half-plane $\sigma>\sigma_{0}$. From this we deduce easily that the series (7) converges uniformly for almost all $\omega \in \Omega$ on compact subsets of $D$. This proves the lemma.

From Lemma 2 it follows that

$$
\widetilde{\varphi}(s, \omega)=\prod_{m=1}^{\infty} \prod_{j=1}^{g(m)}\left(1-\frac{\omega^{f(j, m)}\left(p_{m}\right) a_{m}^{(j)}}{p_{m}^{s f(j, m)}}\right)^{-1}
$$

is an $H(D)$-valued random element defined on the probability space $(\Omega, \mathcal{B}(\Omega), m)$. Denote by $P_{\widetilde{\varphi}}$ the distribution of $\widetilde{\varphi}(s, \omega)$, and let $P_{3, \widetilde{\varphi}}$ be the restriction of $P_{\widetilde{\varphi}}$ to $\left(H\left(D_{3}\right), \mathcal{B}\left(H\left(D_{3}\right)\right)\right)$. Define the probability measure

$$
V_{T, w}(A)=\frac{1}{U} \int_{T_{0}}^{T} w(\tau) I_{\{\tau: \varphi(s+i \tau) \in A\}} d \tau, \quad A \in \mathcal{B}\left(H\left(D_{3}\right)\right) .
$$

THEOREM 8. Under the conditions (1), (2), (4) and (iii), (iv) of Theorem 2 the measure $V_{T, w}$ converges weakly to $P_{3, \widetilde{\varphi}}$ as $T \rightarrow \infty$.

The proof of Theorem 8 is similar to that of Theorem 6 . In this case all lemmas used in the proof of Theorem 6 are one-dimensional ones.

3. Proof of Theorem 7. First we state and prove some results necessary for the proof of Theorem 7 .

Lemma 3. Let $\mathcal{G}$ be a simply connected domain in $\mathbb{C}$. Let $\left\{f_{m}\right\}$ be a sequence in $H(\mathcal{G})$ which satisfies:

(i) if $\mu$ is a complex Borel measure on $(\mathbb{C}, \mathcal{B}(\mathbb{C}))$ with compact support contained in $\mathcal{G}$ such that

$$
\sum_{m=1}^{\infty}\left|\int_{\mathbb{C}} f_{m} d \mu\right|<\infty
$$


then

$$
\int_{\mathbb{C}} s^{r} d \mu(s)=0 \quad \text { for all } r=0,1,2, \ldots ;
$$

(ii) the series $\sum_{m=1}^{\infty} f_{m}$ converges in $H(\mathcal{G})$;

(iii) for any compact $K \subseteq \mathcal{G}$,

$$
\sum_{m=1}^{\infty} \sup _{s \in K}\left|f_{m}(s)\right|^{2}<\infty .
$$

Then the set of all convergent series $\sum_{m=1}^{\infty} a_{m} f_{m}$ with $\left|a_{m}\right|=1$ is dense in $H(\mathcal{G})$.

Pro of. The lemma is a one-dimensional case of Lemma 5.2.9 from [1]; its proof is also given in [7], Theorem 6.3.10.

Let

$$
f_{m}=f_{m}(s)=-\sum_{j=1}^{g(m)} \log \left(1-\frac{a_{m}^{f(j, m)} a_{m}^{(j)}}{p_{m}^{s f(j, m)}}\right)
$$

with $a_{m} \in \gamma$. Here

$$
\log (1+z)=z-\frac{z^{2}}{2}+\frac{z^{3}}{3}-\ldots, \quad|z|<1 .
$$

LEMma 4. Let the condition (5) or (6) be satisfied. Then the set of all convergent series $\sum_{m=1}^{\infty} f_{m}(s)$ is dense in $H\left(D_{3}\right)$.

Pro of. Let $m_{0}$ be a fixed natural number. First we prove that the set of all convergent series

$$
\sum_{m>m_{0}} a_{m} \widetilde{f}_{m}, \quad a_{m} \in \gamma
$$

where

$$
\widetilde{f}_{m}=\widetilde{f}_{m}(s)=-\sum_{j=1}^{g(m)} \log \left(1-\frac{a_{m}^{(j)}}{p_{m}^{s f(j, m)}}\right),
$$

is dense in $H\left(D_{3}\right)$. Let $\left\{\widetilde{a}_{m}: \widetilde{a}_{m} \in \gamma\right\}$ be a sequence such that the series

$$
\sum_{m=1}^{\infty} \widetilde{a}_{m} \widetilde{\widetilde{f}}_{m}
$$

converges in $H\left(D_{3}\right)$. Here

$$
\widetilde{\widetilde{f}}_{m}=\widetilde{\widetilde{f}}_{m}(s)= \begin{cases}\widetilde{f}_{m}, & m \geq m_{0}, \\ 0, & m<m_{0} .\end{cases}
$$


We show that such a sequence $\left\{\widetilde{a}_{m}\right\}$ exists. Clearly,

$$
\widetilde{f}_{m}(s)=\sum_{\substack{j=1 \\ f(j, m)=1}}^{g(m)} \frac{a_{m}^{(j)}}{p_{m}^{s}}+r_{m}(s),
$$

where $r_{m}(s)=B p_{m}^{\alpha+2 \beta-2 \sigma}$ in view of (1), and thus the series

$$
\sum_{m=1}^{\infty} r_{m}(s)
$$

converges uniformly on compact subsets of $D_{3}$. As in the proof of Lemma 2, we find that the series

$$
\sum_{m=1}^{\infty} \frac{\omega\left(p_{m}\right)}{p_{m}^{s}} \sum_{\substack{j=1 \\ f(j, m)=1}}^{g(m)} a_{m}^{(j)}
$$

converges uniformly for almost all $\omega \in \Omega$ on compact subsets of $D_{3}$. Consequently, there exists a sequence $\left\{\widetilde{a}_{m}\right\}$ such that the series

$$
\sum_{m=1}^{\infty} \frac{\widetilde{a}_{m}}{p_{m}^{s}} \sum_{\substack{j=1 \\ f(j, m)=1}}^{g(m)} a_{m}^{(j)}, \quad \widetilde{a}_{m} \in \gamma,
$$

converges in $H\left(D_{3}\right)$. This together with the convergence of the series (10) shows that (9) converges in $H\left(D_{3}\right)$.

Now let $g_{m}=\widetilde{a}_{m} \widetilde{\widetilde{f}}_{m}$. In order to prove the denseness of the set of all convergent series (8) it suffices to show that the set of all convergent series

$$
\sum_{m=1}^{\infty} a_{m} g_{m}, \quad a_{m} \in \gamma,
$$

is dense in $H\left(D_{3}\right)$. For this aim we will verify the hypotheses of Lemma 3. From the definition of $g_{m}$ we know that the hypothesis (ii) of Lemma 3 is satisfied. Moreover, for any compact $K \subset D_{3}$,

$$
\sum_{m=1}^{\infty} \sup _{s \in K}\left|g_{m}(s)\right|^{2}<\infty
$$

that is, the hypothesis (iii) of Lemma 3 is also satisfied. Therefore, it remains to verify the hypothesis (i) of Lemma 3 .

Let $\mu$ be a complex Borel measure with compact support contained in $D_{3}$ such that

$$
\sum_{m=1}^{\infty}\left|\int_{\mathbb{C}} g_{m}(s) d \mu\right|<\infty .
$$


Set

$$
h_{m}(s)=\frac{\widetilde{a}_{m}}{p_{m}^{s}} \sum_{\substack{j=1 \\ f(j, m)=1}}^{g(m)} a_{m}^{(j)} .
$$

Then

$$
\sum_{m=1}^{\infty}\left|g_{m}(s)-h_{m}(s)\right|<\infty
$$

uniformly on compact subsets of $D_{3}$. Consequently, in view of (12),

$$
\sum_{m=1}^{\infty}\left|\int_{\mathbb{C}} h_{m}(s) d \mu\right|<\infty
$$

or

$$
\sum_{m=1}^{\infty}\left|\int_{\mathbb{C}} p_{m}^{-s} \sum_{\substack{j=1 \\ f(j, m)=1}}^{g(m)} a_{m}^{(j)} d \mu\right|<\infty .
$$

Let $D_{3}^{\prime}=\left\{s \in \mathbb{C}: \varrho_{0}^{\prime}<\sigma<1\right\}$ where $\varrho_{0}^{\prime}=\varrho_{0}-(\alpha+\beta) \geq 1 / 2$, and let $h(s)=s-(\alpha+\beta)$. Moreover, let $\mu h^{-1}(A)=\mu\left(h^{-1} A\right), A \in B(\mathbb{C})$. Then $\mu h^{-1}$ is a complex Borel measure with compact support contained in $D_{3}^{\prime}$. Consequently, (13) yields

$$
\sum_{m=1}^{\infty}\left|\int_{\mathbb{C}} \frac{1}{p_{m}^{s+\alpha+\beta}} \sum_{\substack{j=1 \\ f(j, m)=1}}^{g(m)} a_{m}^{(j)} d \mu h^{-1}\right|<\infty,
$$

or

$$
\sum_{m=1}^{\infty}\left|\varrho\left(\log p_{m}\right)\right|\left|p_{m}^{-\alpha-\beta} \sum_{\substack{j=1 \\ f(j, m)=1}}^{g(m)} a_{m}^{(j)}\right|<\infty
$$

where

$$
\varrho(z)=\int_{\mathbb{C}} c^{-s z} d \mu h^{-1}(s) .
$$

Hence, taking into account (5), we deduce that

$$
\sum_{m=1}^{\infty}\left|\varrho\left(\log p_{m}\right)\right|<\infty .
$$

So, we have obtained the same situation as in the proof of Lemma 6.5.4 from [7], and we have

$$
\int_{\mathbb{C}} s^{r} d \mu h^{-1}(s)=0 \quad \text { for all } r=0,1,2, \ldots
$$


Hence we deduce that

$$
\int_{\mathbb{C}} s^{r} d \mu(s)=0 \quad \text { for all } r=0,1,2, \ldots
$$

This shows that the hypothesis (i) of Lemma 3 is satisfied. Now by Lemma 3 we have the denseness of all convergent series (11), and thus of all convergent series (8).

Now let the condition (6) be satisfied. We will prove that, taking into account the change of notation $\left(p_{m} \sim p\right)$,

$$
\sum_{p}|\varrho(\log p)|<\infty
$$

Clearly, by (14) we have

$$
\sum_{\substack{p \\ M(p) \geq \varepsilon}}|\varrho(\log p)|<\infty .
$$

We observe that $\varrho(\log p)=B p^{-\delta_{1}}$ with $\delta_{1}>1 / 2$. Thus, by (6), we find

$$
\sum_{\substack{p \leq x \\ M(\bar{p})<\varepsilon}}|\varrho(\log p)|=B \sum_{\substack{p \leq x \\ M(\bar{p})<\varepsilon}} \frac{1}{p^{\delta_{1}}}=\frac{B \pi(x)}{x^{\delta+\delta_{1}}}+B \int_{2}^{x} \frac{d u}{u^{\delta_{1}+\delta}}=B .
$$

Hence

$$
\sum_{\substack{p \\ M(p)<\varepsilon}}|\varrho(\log p)|<\infty
$$

and this together with (16) implies (15). So, the rest of the proof coincides with that when the condition (5) is used.

Let $x_{0}(s) \in H\left(D_{3}\right), K$ be a compact subset of $D_{3}$ and $\varepsilon>0$. We fix $m_{0}$ such that

$$
\sup _{s \in K}\left(\sum_{m>m_{0}} \sum_{j=1}^{g(m)} \sum_{l=2}^{\infty} \frac{\left|a_{m}^{(j)}\right|^{l}}{l_{m}^{l \sigma f(j, m)}}\right)<\frac{\varepsilon}{4} .
$$

From the denseness of all convergent series (8) we see that there exists a sequence $\left\{\widetilde{a}_{m}: \widetilde{a}_{m} \in \gamma\right\}$ such that

$$
\sup _{s \in K}\left|x_{0}(s)-\sum_{m \leq m_{0}} \widetilde{f}_{m}(s)-\sum_{m>m_{0}} \widetilde{a}_{m} \widetilde{f}_{m}(s)\right|<\frac{\varepsilon}{2} .
$$

Set

$$
a_{m}= \begin{cases}1, & m \leq m_{0} \\ \widetilde{a}_{m}, & m>m_{0}\end{cases}
$$


Then (17) and (18) imply

$$
\begin{aligned}
\sup _{s \in K}\left|x_{0}(s)-\sum_{m=1}^{\infty} f_{m}(s)\right|= & \sup _{s \in K}\left|x_{0}(s)-\sum_{m \leq m_{0}} \widetilde{f}_{m}(s)-\sum_{m>m_{0}} f_{m}(s)\right| \\
\leq & \sup _{s \in K}\left|x_{0}(s)-\sum_{m \leq m_{0}} \widetilde{f}_{m}(s)-\sum_{m>m_{0}} \widetilde{a}_{m} \widetilde{f}_{m}(s)\right| \\
& +\sup _{s \in K}\left|\sum_{m>m_{0}} \widetilde{a}_{m} \widetilde{f}_{m}(s)-\sum_{m>m_{0}} f_{m}(s)\right| \\
< & \frac{\varepsilon}{2}+\sup _{s \in K}\left(2 \sum_{m>m_{0}} \sum_{j=1}^{g(m)} \sum_{l=2}^{\infty} \frac{\left|a_{m}^{(j)}\right|^{l}}{l p_{m}^{l \sigma f(j, m)}}\right)<\varepsilon .
\end{aligned}
$$

Since $x_{0}(s), K$ and $\varepsilon$ are arbitrary, this proves the lemma.

By Theorem 8 the probability measure

$$
\frac{1}{U} \int_{0}^{T} w(\tau) I_{\{\tau: \varphi(s+i \tau) \in A\}} d \tau, \quad A \in \mathcal{B}\left(H\left(D_{3}\right)\right),
$$

converges weakly to the measure $P_{3, \widetilde{\varphi}}$ as $T \rightarrow \infty$ where $P_{3, \widetilde{\varphi}}$ is the distribution of the $H\left(D_{3}\right)$-valued random element

$$
\widetilde{\varphi}(s, \omega)=\prod_{m=1}^{\infty} \prod_{j=1}^{g(m)}\left(1-\frac{\omega^{f(j, m)}\left(p_{m}\right) a_{m}^{(j)}}{p_{m}^{s f(j, m)}}\right)^{-1}, \quad \omega \in \Omega .
$$

Now we will find the support of the measure $P_{s, \widetilde{\varphi}}$. Denote by $S_{X}$ the support of the random element $X$. Let $\mathcal{G}$ be a region in $\mathbb{C}$.

Lemma 5. Let $\left\{X_{m}\right\}$ be a sequence of independent $H(\mathcal{G})$-valued random elements and suppose that the series $\sum_{m=1}^{\infty} X_{m}$ converges almost surely. Then the support of the sum of this series is the closure of the set of all $f \in H(\mathcal{G})$ which may be written as a convergent series

$$
f=\sum_{m=1}^{\infty} f_{m}, \quad f_{m} \in S_{X_{m}} .
$$

Proof. This is Theorem 1.7.10 of [7].

Let

$$
S=\left\{f \in H\left(D_{3}\right): f(s) \neq 0 \text { or } f(s) \equiv 0\right\} .
$$

Lemma 6 . The support of the measure $P_{3, \widetilde{\varphi}}$ is the set $S$.

Proof. The sequence $\left\{\omega\left(p_{m}\right): m \in \mathbb{N}\right\}$ is a sequence of independent random variables. Thus 


$$
\left\{\sum_{j=1}^{g(m)} \log \left(1-\frac{\omega^{f(j, m)}\left(p_{m}\right) a_{m}^{(j)}}{p_{m}^{s f(j, m)}}\right)^{-1}: m \in \mathbb{N}\right\}
$$

is a sequence of independent $H\left(D_{3}\right)$-valued random elements. The support of each $\omega\left(p_{m}\right)$ is the unit circle $\gamma$. Therefore the support of the random elements

$$
\sum_{j=1}^{g(m)} \log \left(1-\frac{\omega^{f(j, m)}\left(p_{m}\right) a_{m}^{(j)}}{p_{m}^{s f(j, m)}}\right)^{-1}, \quad m \in \mathbb{N},
$$

is the set

$$
\left\{f \in H\left(D_{3}\right): f(s)=-\sum_{j=1}^{g(m)} \log \left(1-\frac{a^{f(j, m)} a_{m}^{(j)}}{p_{m}^{s f(j, m)}}\right) \text { with } a \in \gamma\right\} .
$$

Consequently, by Lemma 5, the support of the $H\left(D_{3}\right)$-valued random element

$$
\log \widetilde{\varphi}(s, \omega)=-\sum_{m=1}^{\infty} \sum_{j=1}^{g(m)} \log \left(1-\frac{\omega^{f(j, m)}\left(p_{m}\right) a_{m}^{(j)}}{p_{m}^{s f(j, m)}}\right)
$$

is the closure of the set of all convergent series $\sum_{m=1}^{\infty} f_{m}(s)$. Here we preserve the notation of Lemma 4 . By Lemma 4 the set of these series is dense in $H\left(D_{3}\right)$. The map $h: H\left(D_{3}\right) \rightarrow H\left(D_{3}\right)$ defined by $h(f)=\exp \{f\}$, $f \in H\left(D_{3}\right)$, is a continuous function sending $\log \widetilde{\varphi}(s, \omega)$ into $\widetilde{\varphi}(s, \omega)$ and sending $H\left(D_{3}\right)$ into $S \backslash\{0\}$. Therefore the support of $\widetilde{\varphi}(s, \omega)$ contains $S \backslash\{0\}$. On the other hand, the support of $\widetilde{\varphi}(s, \omega)$ is a closed set. By the Hurwitz theorem (see [14], Section 3.4.5) we have $\overline{S \backslash\{0\}}=S$. Thus

$$
S_{\widetilde{\varphi}} \supseteq S .
$$

Clearly,

$$
\prod_{j=1}^{g(m)}\left(1-\frac{\omega^{f(j, m)}\left(p_{m}\right) a_{m}^{(j)}}{p_{m}^{s f(j, m)}}\right)^{-1}, \quad s \in D_{3}, \omega \in \Omega,
$$

is non-zero for all $m \in \mathbb{N}$. Thus $\widetilde{\varphi}(s, \omega)$ is an almost surely convergent product of non-vanishing factors. Applying the Hurwitz theorem again, we

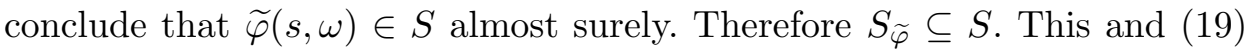
prove the lemma.

Before the proof of Theorem 7 we state the Mergelyan theorem as the following lemma.

Lemma 7. Let $K$ be a compact subset of $\mathbb{C}$ whose complement is connected. Then any continuous function $f(s)$ on $K$ which is analytic in the interior of $K$ is approximable uniformly on $K$ by polynomials in $s$.

The proof is given in [16]. 
Proof of Theorem 7. First we suppose that $f(s)$ has non-vanishing analytic continuation to $H\left(D_{3}\right)$. Denote by $\mathcal{G}$ the set of functions $g \in H\left(D_{3}\right)$ such that

$$
\sup _{s \in K}|g(s)-f(s)|<\varepsilon .
$$

By Lemma 6 the function $f(s)$ is contained in the support $S$ of the random element $\widetilde{\varphi}(s, \omega)$. Since by Theorem 8 the measure $V_{T, w}$ converges weakly to the measure $P_{3, \tilde{\varphi}}$ as $T \rightarrow \infty$ and the set $\mathcal{G}$ is open, we deduce from the properties of weak convergence and support that

$$
\liminf _{T \rightarrow \infty} \frac{1}{U} \int_{T_{0}}^{T} w(\tau) I_{\left\{\tau: \sup _{s \in K}|\varphi(s+i \tau)-f(s)|<\varepsilon\right\}} d \tau \geq P_{3, \widetilde{\varphi}}(\mathcal{G})>0 .
$$

Now let $f(s)$ be as in the statement of the theorem. Then in view of Lemma 7 there exists a sequence $\left\{p_{n}(s)\right\}$ of polynomials such that $p_{n}(s) \rightarrow$ $f(s)$ as $n \rightarrow \infty$ uniformly on $K$. Since $f(s) \neq 0$ on $K$, we have $p_{n_{0}} \neq 0$ on $K$ for sufficiently large $n_{0}$, and

$$
\sup _{s \in K}\left|f(s)-p_{n_{0}}(s)\right|<\varepsilon / 4 .
$$

Since the polynomial $p_{n_{0}}(s)$ has only finitely many zeros, there exists a region $\mathcal{G}_{1}$ whose complement is connected such that $K \subset \mathcal{G}_{1}$ and $p_{n_{0}}(s) \neq 0$ on $\mathcal{G}_{1}$. Thus there exists a continuous version $\log p_{n_{0}}(s)$ on $\mathcal{G}_{1}$, and $\log p_{n_{0}}(s)$ is analytic in the interior of $\mathcal{G}_{1}$. Therefore by Lemma 7 there exists a sequence $\left\{q_{n}(s)\right\}$ of polynomials such that $q_{n}(s) \rightarrow \log p_{n_{0}}(s)$ as $n \rightarrow \infty$ uniformly on $K$. Thus, for sufficiently large $n_{1}$,

$$
\sup _{s \in K}\left|p_{n_{0}}(s)-e^{q_{n_{1}}(s)}\right|<\varepsilon / 4 .
$$

Hence and from (20) we have

$$
\sup _{s \in K}\left|f(s)-\exp \left\{q_{n_{1}}(s)\right\}\right|<\varepsilon / 2 .
$$

From the first part of the proof we deduce that

$$
\liminf _{T \rightarrow \infty} \frac{1}{U} \int_{T_{0}}^{T} w(\tau) I_{\left\{\tau: \sup _{s \in K}\left|\varphi(s+i \tau)-\exp \left\{q_{n_{1}}(s)\right\}\right|<\varepsilon / 2\right\}} d \tau>0 .
$$

This together with (21) proves the theorem.

The author would like to express his deep gratitude to Professor Kohji Matsumoto for careful studying of and comments on the first version of this paper, for indication of some inaccuracies and for suggestions how to improve the results obtained. 


\section{References}

[1] B. Bagchi, The statistical behaviour and universality properties of the Riemann zeta-function and other allied Dirichlet series, Ph.D. thesis, Calcutta, Indian Statistical Institute, 1981.

[2] S. M. Gonek, Analytic properties of zeta and L-functions, Ph.D. thesis, University of Michigan, 1979.

[3] T. Hattori and K. Matsumoto, Large deviations of Montgomery type and its application to the theory of zeta-functions, Acta Arith. 71 (1995), 79-94.

[4] A. Laurinčikas, Limit theorems for the Matsumoto zeta-function, J. Théor. Nombres Bordeaux 8 (1996), 143-158.

[5] - On limit distribution of the Matsumoto zeta-function, Acta Arith. 79 (1997), $31-39$.

[6] -, On limit distribution of the Matsumoto zeta-function. II, Liet. Mat. Rink. 36 (1996), 464-485 (in Russian).

[7] —, Limit Theorems for the Riemann Zeta-Function, Kluwer, Dordrecht, 1996.

[8] A. Laurinčikas and G. Misevičius, On limit distribution of the Riemann zetafunction, Acta Arith. 76 (1996), 317-334.

[9] K. Matsumoto, A probabilistic study on the value-distribution of Dirichlet series attached to certain cusp forms, Nagoya Math. J. 116 (1989), 123-138.

[10] - Value-distribution of zeta functions, in: Lecture Notes in Math. 1434, Springer, 1990, 178-187.

[11] - On the magnitude of asymptotic probability measures of Dedekind zeta-functions and other Euler products, Acta Arith. 60 (1991), 125-147.

[12] —, Asymptotic probability measures of Euler products, in: Proceedings of the Amalfi Conference on Analytic Number Theory (Maiori, 1989), Univ. Salerno, Salerno, 1992, 295-313.

[13] —, Asymptotic probability measures of zeta-functions of algebraic number fields, J. Number Theory 40 (1992), 187-210.

[14] E. C. Titchmarsh, The Theory of Functions, Oxford Univ. Press, Oxford, 1939.

[15] S. M. Voronin, Theorem on the "universality" of the Riemann zeta-function, Izv. Akad. Nauk SSSR Ser. Mat. 39 (1975), 475-486 (in Russian).

[16] J. L. Walsh, Interpolation and Approximation by Rational Functions in the Complex Domain, Amer. Math. Soc. Colloq. Publ. 20, 1960.

Department of Mathematics

Vilnius University

Naugarduko, 24

2006 Vilnius, Lithuania

E-mail: antanas.laurincikas@maf.vu.lt 